\title{
Asymmetric Flankers in Comodulation Masking Release
}

\author{
Akram Pourbakht and Leila Faraji \\ Department of Audiology, School of Rehabilitation Sciences, Iran University of Medical Sciences, Tehran, Iran
}

Received April 8, 2018

Revised May 28, 2018

Accepted June 18, 2018

\author{
Address for correspondence \\ Leila Faraji, MSc \\ Department of Audiology, \\ School of Rehabilitation Sciences, \\ Iran University of Medical Sciences, \\ Tehran, Iran \\ Tel $+98-21-22250541$ \\ Fax +98-21-22220946 \\ E-mail faraji.1@tak.iums.ac.ir
}

\begin{abstract}
Background and Objectives: Detection of auditory signals may be improved when maskers far from the frequency of the target signal are coherently amplitude-modulated. This improvement of signal detection is called comodulation masking release (CMR). In the CMR experiments, flankers have been usually arranged symmetrically. In practice, we will be confronted with a problem by using symmetric flankers due to the limited output of clinical audiometers, especially at high-frequency. We aimed to check whether flanker arrangement has any effect on the amount of CMR, especially when there is no flankers with a frequency higher than the signal. Subjects and Methods: Eighteen normal hearing listeners ranging in age from 20 to 46 years old participated. Symmetric (2-2) and asymmetric (3-1 and 4-0) flankers were used and then the amount of CMR compared among them. Results: Our results showed in the same numbers of flankers, there were no statistically CMR differences between symmetric and asymmetric arrangement. Also when we did not have a flanker at a frequency higher than the signal and all flankers were placed below the signal, there was no statistically difference with the symmetric arrangement. Conclusions: The asymmetry of the flankers and also omitting the flankers with a frequency higher than the signal, have no effect on CMR results. We concluded that CMR can be considered by using clinical audiometer.
\end{abstract}

J Audiol Otol 2019;23(1):27-32

KEY WORDS: Comodulation Masking Release · Asymmetric flanker · Symmetric flanker.

\section{Introduction}

A target signal that setting in another masking sound can be better detected by adding energy that is remote in frequency from both the masker and target signal [1]. This effect is known as comodulation masking release (CMR) and is observed when the remote sound and the masker share coherent patterns of amplitude modulations [1-6]. CMR is measured in two ways. The first (earlier method) often referred to as the "band-widening experiment," is to use a single band of noise, centered around the signal frequency, as a masker, and to compare thresholds for modulated and unmodulated noise maskers as a function of the masker bandwidth. The second method (preferential method) is to use a masker consisting of several narrow masker bands of noises, typically with bandwidths between 20 and $50 \mathrm{~Hz}$, which have relatively slow inherent

This is an Open Access article distributed under the terms of the Creative Commons Attribution Non-Commercial License (https://creativecommons.org/licenses/by-nc/4.0/) which permits unrestricted non-commercial use, distribution, and reproduction in any medium, provided the original work is properly cited. amplitude modulation; one band is centered at the signal frequency (on-frequency masker) and one or more other bands (flanking bands or off-frequency maskers) are spectrally separated from the signal frequency [2].

CMR has been investigated in many studies and most of them used the second method. In most of them, the arrangement of the flankers is considered symmetric and asymmetric arrangement of flankers is less used. Two reviews that were somewhat similar to ours, related to Yasin, et al. [7] and Dau, et al. [1]. Yasin, et al. [7] examined CMR in two arrangement; with and without flankers. Their results showed no significant difference in masking release results between in two situation, existence or absence of flankers (there was on-frequency masker). In the review of Dau, et al. [1], after the high-frequency flankers, a multi-flanker was added as postcursor and CMR measured in two arrangements. Their results also showed no significant difference in two arrangements. In Dau, et al's study [1] which was an interesting study, the symmetrical arrangement of flankers was also used and examined the effect of perceptual grouping mechanisms on CMR. The results are 
consistent with the idea that modulation analysis occurs within, not across, auditory objects, and that across-frequency CMR only occurs if the on-frequency and flanking bands fall within the same auditory object or stream [7-11]. In the evaluation of CMR with symmetric flankers at a high-frequency signal, there is a limitation in output of clinical audiometers for the high-frequency flankers. Because most audiometers are not capable to have frequency $>12,000 \mathrm{~Hz}$, therefore CMR evaluation at higher frequencies is not available and this explain why CMR has not been tested at the frequencies higher than 8-10 kHz. In the CMR usually the flankers are symmetrically in the up and down to the signal. For example in the CMR review at 3,000 $\mathrm{Hz}$ (as signal), the low-frequency flankers are 2,000 Hz and 1,000 Hz. While high-frequency flankers include $4,000 \mathrm{~Hz}$ and $6,000 \mathrm{~Hz}$. There will be no problem in this regard. Now if we want to check the CMR at $8,000 \mathrm{~Hz}$ (as signal), there will be no problem in generation of low-frequency flankers (i.e., 4,000 Hz and 2,000 Hz or 6,000 $\mathrm{Hz}$ and $12,000 \mathrm{~Hz}$ ). But the high-frequency flankers in this case will be $16,000 \mathrm{~Hz}$ and $32,000 \mathrm{~Hz}$, which clinical audiometers can not product them. Considering the asymmetry of traveling wave in the cochlea at the higher frequencies (in the base of cochlea) and the lack of effect of them on the lower frequencies (in the apex of cochlea), the question is whether limiting the number of high-frequency flankers has any effect on CMR results or not? According to Fletcher theory, there are many filters in cochlea that each one had a shape. Considering to the shape of auditory filters and their position of them in cochlea (high in base and low in apex of the cochlea), we assumed that the symmetry or asymmetry of the flankers is not affected CMR results. In fact due to location and the asymmetric shape of auditory filters at high-frequency (base of cochlea) it seems that the high-frequency tone not affected the low-frequency tone (apex of cochlea) that is present at the same time. Critical band of filters specified with equivalent rectangular bandwidth (ERB) and ERB in low and highfrequency is completely difference. Therefore a high-frequency tone did not marked effect on low-frequency tone, because ERB in both of them did not has any overlapping [12-14]. Our main assumption in this study was that symmetry or asymmetry of flankers have no effect on CMR results. For that, we aimed in this study to measure CMR by using asymmetric flankers and comparing the results to the symmetric-flankers measured CMR.

\section{Subjects and Methods}

\section{Participants}

Eighteen normal hearing listeners ranging in age from 20 to 46 years old participated in this study. They were randomly selected among students and staff of audiology clinic of the School of Rehabilitation Sciences of Iran University of Medical Sciences. The behavioral pure-tone audiometry thresholds levels were measured to be than $20 \mathrm{~dB}$ HL in octave frequencies of $250-8,000 \mathrm{~Hz}$. Also, impedance tests (tympanometry and refletometry) were done for all case. All of persons were considered to have normal levels of memory and cognitive functions according to the Wechsler test and Mini-Mental State Examination test, which is a critical assumption for performing the CMR experiment.

The subjects signed an informed consent form before the experimental process began. All procedures were approved by the Institutional Review Board of Iran University of Medical Sciences (\#IR.IUMS.REC1396.0457).

\section{Equipments and stimuli}

In this study, we used Orbiter922 Audiometer (Madsen, Taastrup, Denmark), Clarinet plus printer Inventis tympanometer (Inventis, Padova, Italy) for assessment of hearing and middle ear function. The listeners were in a soundproof booth and stimuli were presented diotically via Sennheiser HDA200 headphone (Sennheiser, Old Lyme, CT, USA). The signal (and maskers) generation and presentation during the experiments were controlled by laptop and audiometer using the software package for MATLAB (2013a, Mathworks, Inc., Natick, MA, USA). In this experiment, stimuli consisted of a warble tone (as a target) and narrowband noises (as maskers). In this study, we used a $6,000 \mathrm{~Hz}$ as signal because CMR is high in high frequency signal $[1,2,7]$. The carrier frequency of the warble tone was $5 \mathrm{kHz}$ with a frequency deviation of $15 \%$. The intensity of the signal was $60 \mathrm{~dB}$ with duration of $250 \mathrm{~ms}$. There were one on-frequency masker and four narrow band noise maskers as flankers (or off-frequency maskers), typically with bandwidths $50 \mathrm{~Hz}$. The spacing of flankers toward the target, on-frequency masker and to each other was half an octave. The CMR is high when: 1) the total masker's bandwidth is large, 2) the modulation frequency is low, 3) the modulation depth is high, 4) the envelope is regular, and 5) the masker's spectrum level is high [1-3,7]. Therefore the modulation frequency was $40 \mathrm{~Hz}$ with a modulation depth of $0.8 \%$. The intensity of the on-frequency masker and flankers was $60 \mathrm{~dB}$ with duration of $300 \mathrm{~ms}$. Duration of maskers was considered to be longer than the signal, in order to prevent the occurrence of overshoot [15]. These feature of signal and maskers were according to previous studies [7-10]. The arrangement of flankers has been shown in Fig. 1. 
Fig. 1. A flow chart for the experimental conditions of three flankers.
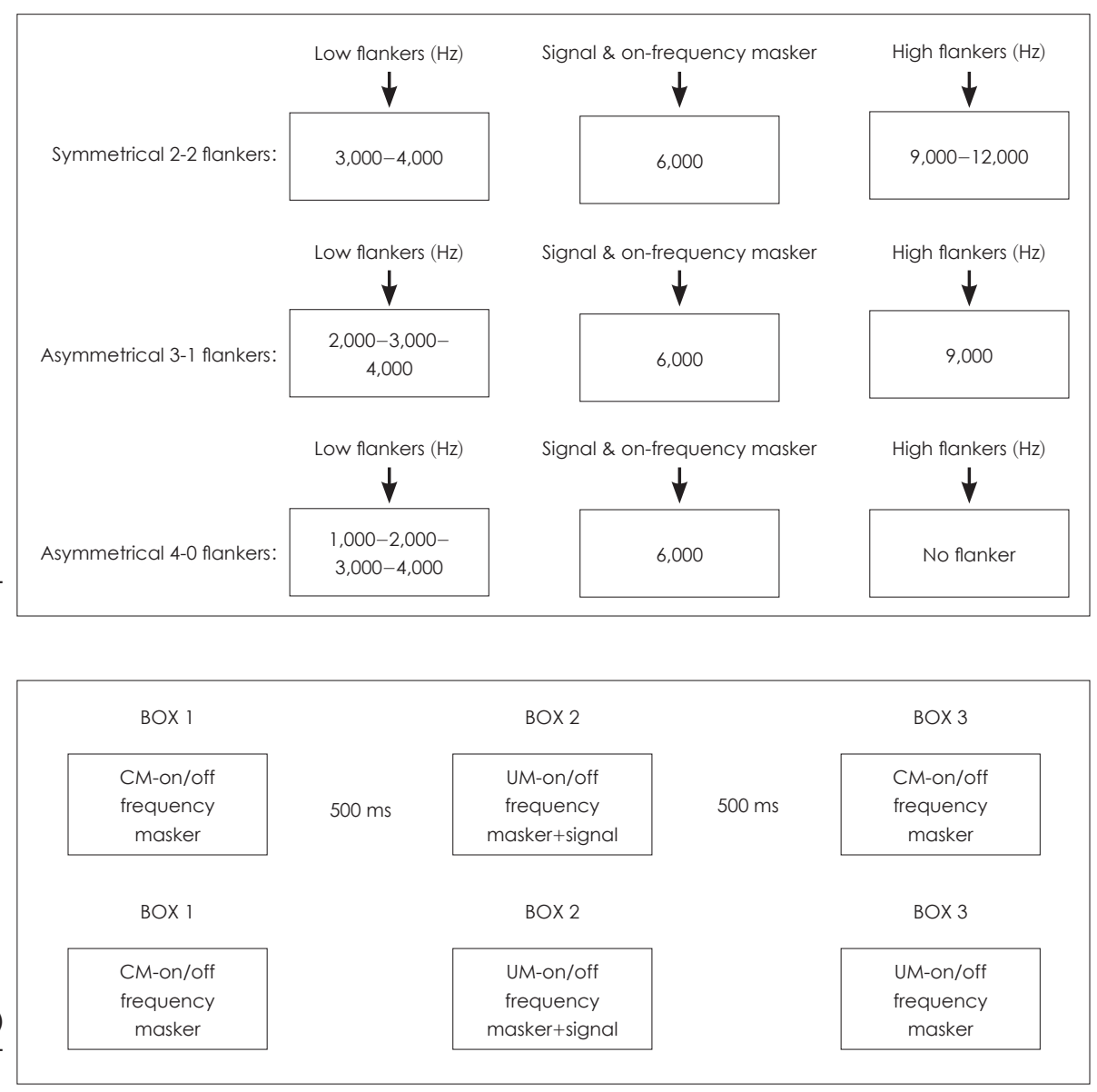

Fig. 2. Detection of signal in $\mathrm{CM}$ (top) and UM (down) maskers. CM: Comodulation, UM: unmodulation.

\section{Experimental procedures}

After performing audiometry and impedance tests, as well as memory and cognitive tests, the CMR was assessed. To obtain CMR (for each arrangement of flankers), first we obtained the threshold of signal in presence of maskers without amplitude modulation (unmodulation, UM). Then we determined the threshold of signal in presence of maskers with amplitude modulation (comodulation, CM). After that, the CMR was calculated by subtracting the signal threshold in presence of the maskers without amplitude modulation (UM) from signal threshold in presence of the maskers with amplitude modulation $(\mathrm{CM})$. That is $\mathrm{CMR}=\mathrm{UM}-\mathrm{CM}$.

At this study, there were three box (or three group of maskers) with $500 \mathrm{~ms}$ between each others [7-10]. In unmodulation situation (UM), content of three boxes include of two unmodulation of maskers and one comodulation of maskers and signal was randomly placed in the box that was included of unmodulation of maskers. In comodulation situation (CM), content of three boxes include of two comodulation of maskers and one unmodulation of maskers and signal was randomly placed in the box that was included of comodulation of maskers (Fig. 2). All signal detections and thresholds were performed using a three-alternative forced choice procedure with adaptive signal level adjustment. Each trial was contained three intervals with a spacing of $500 \mathrm{~ms}$ [16-19]. The signal was randomly placed to an interval, and the person should said which of the intervals contained the signal. The signal intensity was adjusted according to the two-down, one-up procedure to estimate $70.7 \%$ of the psychometric point. The initial step size of the target level was $4 \mathrm{~dB}$, which was reduced to $2 \mathrm{~dB}$ and $1 \mathrm{~dB}$ after the second and third reversals, respectively. When we arrived at step size $1 \mathrm{~dB}$, the trend continued up to six reversals and then the average of last six reversals was calculated and considered as threshold estimate. Three threshold estimates were obtained and an average of them was considered as a real threshold. In unmodulation position, the content of three intervals were of two unmodulated and one comodulated maskers and signal was randomly placed on unmodulated maskers. In the comodulated situation, the content of three intervals were of two comodulated and one unmodulated maskers and signal was randomly placed on comodulated maskers.

In each trial, there were three intervals and subjects asked to detect the interval containing the signal (warble tone). The lis- 
teners responded verbally and the true answer was confirmed while the listener was informed when giving false answer.

In the statistical analysis, Kolmogorov-Smirnov test was utilized for normal distribution of CMR findings and then paired t-test was used to compare the CMR means in each pair of flankers (2-2, 3-1, and 4-0 flankers).

\section{Results}

Statistical analysis was conducted in SPSS software ver. 20 (IBM Corp., Armonk, NY, USA). The Kolmogorov-Smirnov test indicated that all data followed a normal distribution ( $p>$ 0.05 ). We evaluated eighteen (male: 9 , female: 9) participants from 20 to 46 years old (mean: 31.83 , SD: 7.77). All participants had normal cognition, memory and impedance tests (normal type of tympanometry \& reflectometry). The behavioral pure-tone audiometry thresholds levels in all persons in 6,000 Hz were less than $15 \mathrm{~dB}$ (mean: 5, SD: 3.24). The assessments of CMR were done on the right ear. Mean of UM thresholds in 2-2 flankers was $67.13 \mathrm{~dB}$ (SD: 4.12). While mean of CM thresholds in 2-2 flankers was $55.47 \mathrm{~dB}$ (SD: 3.62). In asymmetric 3-1 flankers, mean of UM thresholds was $69.12 \mathrm{~dB}$ (SD: 5.31 ) and mean of $\mathrm{CM}$ thresholds was $57.10 \mathrm{~dB}$ (SD: 4.68). Mean of UM thresholds in 4-0 flankers was $65.27 \mathrm{~dB}$ (SD: 3.16) while mean of $\mathrm{CM}$ thresholds was $53.67 \mathrm{~dB}$ (SD: 3.38) (Fig. 3). When UM thresholds were compared with $\mathrm{CM}$ thresholds in each arrangement flankers, the paired t-test results revealed no significant differences between means of UM $(p=0.63)$ and CM $(p=0.51)$ for the 3-1 flankers (asymmetric flankers) with 2-2 flankers (symmetric flankers). Also, comparison of means of UM ( $p=0.46)$ and CM $(p=0.58)$ thresholds for the 4-0 flankers with 2-2 flankers, did not show any significant difference. The comparisons of the means of UM and CM thresholds between 3-1 and 4-0 flankers, did not show significant difference $(p=0.43$ $p=0.61$, respectively).

CMR for each of the eighteen listeners was calculated separately for each the flanker arrangement in 6,000 Hz. The CMR mean with 2-2 flankers was $11.97 \mathrm{~dB}$ (SD: 3.27), while in the 3-1 and 4-0 flankers, was respectively $12.05 \mathrm{~dB}$ and $11.60 \mathrm{~dB}$ (SD: 3.28 and SD: 2.96) (Table 1). The paired t-test results revealed no significant differences between the flankers arrangement. In comparison of 2-2 and 3-1 flankers CMR, the $p$-value and degree of freedom (df) were 0.87 and

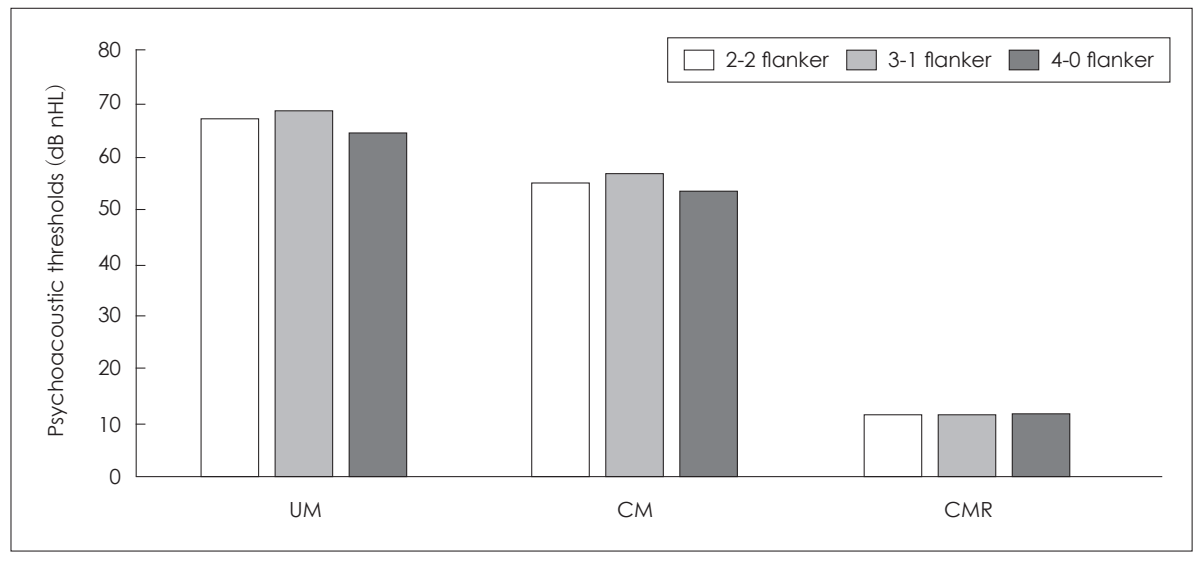

Fig. 3. Comparison of average psychoacoustic thresholds of signal for unmodulated (UM) and comodulated (CM) maskers and comodulation masking release (CMR) value in participants. Y-axis is measured by $\mathrm{dB}$ $\mathrm{nHL}$.

Table 1. Average of $\mathrm{CMR}(\mathrm{dB} \mathrm{nHL})$ in three arrangement of flankers

\begin{tabular}{lccc}
\hline & CMR: symmetric flankers (2-2) & CMR: asymmetric flankers (3-1) & CMR: asymmetric flankers (4-0) \\
\hline Mean & 11.97 & 12.05 & 11.60 \\
SD & 3.27 & 3.28 & 2.96 \\
\hline
\end{tabular}

SD: standard deviation, CMR: comodulaton masking release

Table 2. Paired sample test results for the three arrangement of flankers

\begin{tabular}{|c|c|c|c|c|c|c|c|c|}
\hline & \multicolumn{5}{|c|}{ Paired difference } & \multirow{3}{*}{ t-value } & \multirow{3}{*}{ df } & \multirow{3}{*}{$\begin{array}{c}\text { Sig. } \\
\text { (2-tailed) }\end{array}$} \\
\hline & \multirow{2}{*}{ Mean } & \multirow{2}{*}{ SD } & \multirow{2}{*}{ SEM } & \multicolumn{2}{|c|}{$95 \% \mathrm{Cl}$ of the difference } & & & \\
\hline & & & & Lower & Upper & & & \\
\hline 2-2 vs. 3-1 flankers CMR & -0.08389 & 2.1795 & 0.5137 & -1.1677 & 0.9999 & -0.163 & 17 & 0.872 \\
\hline 2-2 vs. 4-0 flankers CMR & 0.37111 & 1.4874 & 0.3505 & -0.3685 & 1.1109 & 1.059 & 17 & 0.305 \\
\hline 3-1 vs. 4-0 flankers CMR & 0.45500 & 1.6931 & 0.3990 & -0.3869 & 1.2969 & 1.140 & 17 & 0.270 \\
\hline
\end{tabular}

CMR: comodulaton masking release, SD: standard deviation, $\mathrm{Cl}$ : confidence interval, SEM: standard error of the mean, df: degree of freedom 
17 , respectively. The $p$-value and $\mathrm{df}$ were 0.30 and 17 when we compared of 2-2 and 4-0 flankers CMR mean. In comparison of means CMR between the 3-1 flankers and 4-0 flankers, were not seen significant differences ( $p=0.27$, df: 17) (Table 2).

\section{Discussion}

Due to the crucial role of flankers in producing CMR, one important question is proposed; "Is arrangement of flankers has any effect on CMR results? Or, do flankers with more high-frequency (toward the target) have any effect on the detection of the target? To answer this question we used three arrangements of the flankers (one symmetric \& two asymmetric) to investigate the probable effect of higher frequency flankers on CMR results. Our main assumption in this study was that symmetry or asymmetry of flankers have no effect on CMR results. There has not been a study about the role of symmetric or asymmetric of flankers, which in fact was the innovation of our research. The only two studies that were someone similar to our study, related to Yasin, et al. [7] and Dau, et al. [1]. Yasin, et al. [7] investigated masking release in two conditions with and without flankers (there was on-frequency masker in both conditions). Their results showed that there was not significant difference between the results of masking release in two conditions. Dau, et al. [1] added a multiflanker after the high-frequency flankers, as postcursors, and CMR measured in two arrangements (with and without postcursors after high-frequency flankers). Their results also showed no significant difference in two arrangements in CMR results. In fact, their results are consistent with the idea CMR function is a within-channel processing. The CMR experiments using flanking bands pointed out that it is imprecise to assume that one channel is receiving only the on-frequency band plus signal and another channels is receiving only the flanking bands $[1,2]$. Often, the bands will be incompletely resolved. When this happens, the resulting waveform may contain envelope fluctuations resulting from beats between the carrier frequencies of the on-frequency and the flanker bands. These beats can facilitate signal detection without across-channel comparisons being involved. Thus, the CMR in many situations can be explained in terms of the use of within-channel rather than across-channel cues [2]. Therefore, the general content of frequency is important. The results of present study also showed that the use of flankers with higher frequency than to signal did not affect the CMR results and CMR results in three arrangements have not significant differences.

On the other, the traveling wave is most important stage in the analysis of sound in the auditory system. The patterns and positions of the wave depend on the frequency of the stimu- lus and create a peak that is limited to narrow region of the basilar membrane in the cochlea which is defined as critical band or ERB. In the cochlea, there are several filters, each of which has a certain bandwidth that is specified with ERB. ERBs in high-frequencies are also lower than low-frequency. For example, ERB (or critical band) in $100 \mathrm{~Hz}$ is $35 \%$ of the frequency but at $10,000 \mathrm{~Hz}$ is $11 \%$ of the frequency $[6,13]$. Traveling wave in low and high-frequency regions does not have the same shape. The shape of traveling wave around the characteristic frequency in low and high-frequency are symmetric and asymmetric, respectively $[6,13]$. The peak of stimuli with low-frequency is in the apex and peak of stimuli with high frequency is at the base of the cochlea. Then, low-frequency sounds propagate a long way along the membrane, and high-frequency sounds travel only a short way along. In fact, due to the location of higher frequencies in the cochlea (base), the short distance they travel in the cochlea as well as small ERB (compared with low frequency), they have a negligible effect (or even no effect) on the masking of low-frequencies $[15,20,21]$. Just as seen in the results, there were not significant differences in the three flankers' arrangements in CMR values. This means that the high-frequency flankers did not have any effect on the target detection since auditory filters in the high-frequency do not significant effect on the low-frequency tracing and waveform of signal and maskers incompletely resolved. This resulting waveform contain envelope fluctuations resulting from beats between the carrier frequencies of the on-frequency and the flanker bands. These beats can facilitate signal detection, which does not correlate with the frequency details of the resulting waveform. The overall content of the frequency relevant to signal and surrounding areas is important $[2,8,10]$.

According to the results of the present study, in the evaluation of CMR in high-frequency signal, the use of high-frequency flankers than signal can be limited or even eliminated. This result are very important and has a practical application when we conduct CMR by using a clinical audiometer which is limited in the high-frequencies output. Because most clinical audiometer are capable of producing high-frequencies up to $12,000 \mathrm{~Hz}$ (maximum). Therefore, CMR measurement in $8,000 \mathrm{~Hz}$ is impossible, because high-frequency flankers will be $16,000 \mathrm{~Hz}$ and $32,000 \mathrm{~Hz}$.

There were limitations to this study. Our limitations were the lack of evaluations in higher-frequencies (due to our audiometer was limit in higher-frequencies output) and lower frequencies (time limitation) than $6,000 \mathrm{~Hz}$. It is recommended CMR evaluation be done in higher and lower signals to compared results. 


\section{Acknowledgments}

The authors would like to thank from Dr. Samer Mohsen and anonymous reviewer for their very helpful and supportive comments.

\section{Conflicts of interest}

The authors have no financial conflicts of interest.

\section{REFERENCES}

1) Dau T, Ewert S, Oxenham AJ. Auditory stream formation affects comodulation masking release retroactively. J Acoust Soc Am 2009; 125:2182-8.

2) Verhey JL, Pressnitzer D, Winter IM. The psychophysics and physiology of comodulation masking release. Exp Brain Res 2003;153: 405-17.

3) Carlyon RP, Buus S, Florentine M. Comodulation masking release for three types of modulator as a function of modulation rate. Hear Res 1989;42:37-45.

4) Buss E, Hall JW 3rd. Factors contributing to comodulation masking release with dichotic maskers. J Acoust Soc Am 2008;124:1905-8.

5) Rostami S, Moossavi A. Musical training enhances neural processing of comodulation masking release in the auditory brainstem. Audiol Res 2017;7:185

6) Pierzycki RH, Seeber BU. Comodulation masking release in electric hearing. J Assoc Res Otolaryngol 2014;15:279-91.

7) Yasin I, Fantini DA, Plack C. Effect of flanker band number and excitation pattern symmetry on envelope comparisons in masking release. J Acoust Soc Am 2006;120:3084-91.

8) Verhey JL, Dau T, Kollmeier B. Within-channel cues in comodulation masking release (CMR): experiments and model predictions using a modulation-filterbank model. J Acoust Soc Am 1999;106: 2733-45.

9) Grose JH, Hall JW 3rd, Buss E. Across-channel spectral processing. Int Rev Neurobiol 2005;70:87-119.
10) Grose JH, Buss E, Hall JW 3rd. Within- and across-channel factors in the multiband comodulation masking release paradigm. J Acoust Soc Am 2009;125:282-93.

11) Goldman SA, Baer T, Moore BC. Within-channel cues to comodulation masking release for single and symmetrically placed pairs of flanking bands. J Acoust Soc Am 2010;128:2988-97.

12) Neuert V, Verhey JL, Winter IM. Responses of dorsal cochlear nucleus neurons to signals in the presence of modulated maskers. J Neurosci 2004;24:5789-97.

13) Pickles JO. The cochlea. In: Pickles JO, editor. An Introduction to the Physiology of Hearing. 3rd ed. United Kingdom: Emerald Group Publishing limited;2008. p.25-70.

14) Moore DR. Frequency selectivity and masking. In: Moore DR, editor. The Oxford Handbook ofAuditory Science: Hearing. Vol.3. 1st ed. New York: Oxford University Press Inc;2010. p.5-44.

15) Gelfand SA. Hearing, an introduction to psychological and physiological acoutics. 4th ed. New York: Marcel Dekker;2005. p.1-588.

16) Levitt H. Transformed up-down methods in psychoacoustics. J Acoust Soc Am 1971;49:Suppl 2:467-77.

17) Schlauch RS, Rose RM. Two-, three-, and four-interval forcedchoice staircase procedures: estimator bias and efficiency. J Acoust Soc Am 1990;88:732-40.

18) Shelton BR, Scarrow I. Two-alternative versus three-alternative procedures for threshold estimation. Percept Psychophys 1984;35:38592.

19) Ulrich R, Miller J. Threshold estimation in two-alternative forcedchoice (2AFC) tasks: the Spearman-Kärber method. Percept Psychophys 2004;66:517-33.

20) Gorga MP, Neely ST, Dierking DM, Kopun J, Jolkowski K, Groenenboom K, et al. Low-frequency and high-frequency cochlear nonlinearity in humans. J Acoust Soc Am 2007;122:2172-90.

21) Salt AN, Hullar TE. Responses of the ear to low frequency sounds, infrasound and wind turbines. Hear Res 2010;268:12-21. 